\title{
Telehealth Management of Parkinson's Disease Using Wearable Sensors: An Exploratory Study
}

\author{
Dustin A. Heldman ${ }^{a}$ Denzil A. Harris ${ }^{b, c}$ Timothy Felong ${ }^{c}$ \\ Kelly L. Andrzejewskid, e E. Ray Dorseyc, d Joseph P. Giuffrida ${ }^{\text {a }}$ \\ Barry Goldberg ${ }^{a}$ Michelle A. Burack ${ }^{d}$ \\ ${ }^{a}$ Great Lakes NeuroTechnologies Inc., Cleveland, OH, USA; bSchool of Medicine and \\ Dentistry, University of Rochester Medical Center, Rochester, NY, USA; ${ }^{C}$ Center for Human \\ Experimental Therapeutics, University of Rochester Medical Center, Rochester, NY, USA; \\ ${ }^{\mathrm{d}}$ Department of Neurology, University of Rochester Medical Center, Rochester, NY, USA; \\ e Department of Neurology, Jacobs School of Medicine and Biomedical Sciences, \\ University at Buffalo, Buffalo, NY, USA
}

\section{Keywords}

Telehealth management $\cdot$ Parkinson's disease $\cdot$ Wearable sensors

\section{Abstract}

Background: Parkinson's disease (PD) motor symptoms can fluctuate and may not be accurately reflected during a clinical evaluation. In addition, access to movement disorder specialists is limited for many people with PD. The objective of this study was to assess the impact of motion sensor-based telehealth diagnostics on PD clinical care and management. Methods: Eighteen adults with PD were randomized to control or experimental groups. All participants were instructed to use a motion sensor-based monitoring system at home 1 day per week for 7 months. The system included a finger-worn motion sensor and tablet-based software interface that guided patients through tasks to quantify tremor, bradykinesia, and dyskinesia. Data were processed into motor symptom severity reports, which were reviewed by a movement disorder neurologist for the experimental group participants. After 3 months and 6 months, the control group participants visited the clinic for a routine appointment, while the experimental group participants had a videoconference or phone call instead. Results: Homebased assessments were completed with a median compliance of $95.7 \%$. For a subset of participants, the neurologist successfully used information in the reports, such as quantified responses to treatment or progression over time, to make therapy adjustments. Changes in clinical characteristics from study start to end were not significantly different between the groups. Discussion: Individuals with PD were able and willing to use remote monitoring tech- 
nology. Patient management aided by telehealth diagnostics provided comparable outcomes to standard care. Telehealth technologies combined with wearable sensors have the potential to improve care for disparate PD populations or those unable to travel.

\section{Introduction}

Management of Parkinson's disease (PD) symptoms by a neurologist or movement disorder specialist helps maintain a high standard of care; however, access is limited for a significant proportion of this patient population [1]. Telehealth technologies that improve access for patients can have a significant impact on equity, accessibility, and management for patients in rural and remote communities or those unable to travel [2-8]. Clinicians typically use rating scales such as the Unified Parkinson's Disease Rating Scale (UPDRS) [9] to evaluate and fine-tune therapeutic interventions for PD. However, such assessments require access to well-trained clinicians, which can limit effective symptom management for many PD patients [10]. In addition, rating scales only obtain an infrequent snapshot of symptoms in an artificial clinical environment.

Telehealth technologies for PD include tools for objective ratings of symptoms (e.g., motion sensors or surface electromyography) and systems for remote communications with healthcare providers (e.g., videoconferencing) [11]. A number of research and commercial systems have been developed to obtain accurate and objective measures of PD symptoms including tremor, bradykinesia, gait, speech, and dyskinesia [12-25]. Wearable sensors can provide high temporal and spatial resolution, which can be beneficial for capturing symptoms that change over time. Studies have shown a high acceptance and usage compliance of wearable sensors by individuals with PD [26-28]. A recent study demonstrated that homebased motor monitoring can provide cost-effective clinical information and help enhance care for patients with advanced PD [6]. Likewise, studies have shown live videoconferencing may be able to provide similar clinical benefit to in-person care for patients with PD [3, 29-31].

The goals of this study were to assess and evaluate the impact of a telehealth system that includes objective home-based motor assessment, clinically validated motor symptom quantification algorithms, color-coded symptom reporting, and live videoconferencing with a clinician.

\section{Methods}

We conducted a 7-month randomized study of individuals with PD clinically diagnosed by a movement disorder specialist. Individuals were primarily recruited from the movement disorder clinic at the University of Rochester Medical Center with supplemental recruitment occurring from local PD clinics in the Western New York region. Eligible study participants were required to have a score $>22$ on the Montreal Cognitive Assessment (MoCA) scale [32] and could not have or be scheduled to receive deep brain stimulation surgery during their enrollment in the study. The study was reviewed and approved by the Institutional Review Board at the University of Rochester and completed in accordance with the Declaration of Helsinki. All participants provided signed informed consent prior to enrollment.

During a baseline visit, all participants were randomized to control or experimental groups and completed a series of questionnaires including the 39-item Parkinson's Disease Questionnaire (PDQ-39) [33], the Patient Assessment of Chronic Illness Care (PACIC) [34], 
and the 13-item Patient Activation Measure (PAM-13) [35]. A neurologist (K.L.A.) at the University of Rochester, blinded to group assignment, performed an MDS-UPDRS evaluation. Each participant then had an initial evaluation with the treating neurologist (M.A.B.). Responses to all questionnaires and storage of all study-related data were managed using secure, web-based REDCap electronic data capture tools hosted at the University of Rochester [36].

At the conclusion of the baseline visit, all participants were provided with, trained on, and instructed to use an objective motion sensor-based monitoring system (Kinesia ${ }^{\mathrm{TM}}$, Great Lakes NeuroTechnologies Inc., Cleveland, OH, USA) at home 1 day per week for 7 months to perform motion sensor-based motor assessments. The system included a wireless fingerworn motion sensor and touchscreen tablet interface that guided patients through standardized tasks to quantify PD motor features. On the assessment day, the system alerted the participant to perform a motor assessment 6 times per day, $2 \mathrm{~h}$ apart, beginning in the morning before taking any PD medication. The Kinesia systems were programmed to instruct the participant to wear the motion sensor on the index finger of the hand on the more parkinsonian side and guide the participant through the motor assessment. For this study, each motor assessment consisted of $15 \mathrm{~s}$ of arms resting to evaluate resting tremor and dyskinesias, $15 \mathrm{~s}$ of arms extended to evaluate postural tremor and dyskinesias, and $15 \mathrm{~s}$ of repetitive finger tapping to evaluate bradykinesia (speed), hypokinesia (amplitude), and dysrhythmia (rhythm). Motion data from the sensor were uploaded via mobile broadband to a secure cloud server for processing into 0-4 scores previously shown to be highly correlated with clinician ratings $[12,14,17]$ and responsive to therapy changes $[37,38]$. Compliance in using the Kinesia system at home was defined as the percentage of weeks a participant completed at least 6 Kinesia assessments on at least 1 day during the week.

All participants used the system to avoid placebo effects; however, the treating neurologist was given access via a web-interface to view motor symptom severity reports solely for individuals in the experimental group. The reports displayed color-coded motor symptom severities throughout the day as well as the daily averages and standard deviations. The neurologist used these reports to aid in therapy management decisions. Participants in the control group returned to the clinic after 3 months and again after 6 months for a routine appointment. Instead of visiting the clinic, individuals in the experimental group teleconferenced (videoconference or phone call) with the neurologist after 3 and 6 months. At the start of the study, videoconferencing was integrated in the Kinesia platform; however, this feature was removed about midway through the study due to various technical problems including institutional firewalls, mobile broadband connectivity at participants' homes, and difficulties in maintaining videoconferencing software integrated in the Kinesia platform, after which phone calls were used instead. All patient-provider communications and therapy changes were documented. After month 7, all participants returned to the clinic and completed the same questionnaires and assessments completed during the baseline visit as well as a questionnaire on the usability of the Kinesia system.

Compliance, time spent with the neurologist, UPDRS scores, patient questionnaire responses, and Kinesia metrics were examined as outcome measures. Nonparametric tests were used for statistical analyses since normality could not be assumed based on the small sample size. Within-group comparisons of metrics from study start to end were performed using a Wilcoxon signed-rank test, whereas comparisons between the two groups were performed using a Mann-Whitney $U$ test. Kinesia metrics for the study start and end were computed separately for each symptom using outputs from the home-based motor assessments. The study start included average scores from the first 5 weeks of assessments, whereas the study end included average scores from the final 5 weeks. These assessments were performed 1 day per week, 6 times per day; however, the first assessment in the morning each 
Table 1. Baseline characteristics of the study population

\begin{tabular}{lcc}
\hline & Experimental group $(n=9)$ & Control group $(n=9)$ \\
\hline Males/Females, $n$ & $5 / 4$ & $7 / 2$ \\
Mean age \pm SD, years & $65.2 \pm 10.1$ & $68.6 \pm 10.2$ \\
Mean disease duration \pm SD, years & $4.8 \pm 3.5$ & $5.9 \pm 3.7$ \\
Mean levodopa equivalent daily dose \pm SD, mg & $601 \pm 310$ & $858 \pm 392$ \\
\hline
\end{tabular}

Table 2. Changes in clinical and questionnaire metrics

\begin{tabular}{|c|c|c|c|c|c|c|}
\hline & \multicolumn{2}{|l|}{ Baseline } & \multicolumn{2}{|l|}{ Study end } & \multicolumn{2}{|l|}{ Change from baseline } \\
\hline & experimental group & control group & experimental group & control group & experimental group & control group \\
\hline UPDRS $1 \mathrm{~A}$ & $1.0(0.0$ to 3.0$)$ & $2.0(0.0$ to 4.3$)$ & $1.0(0.8$ to 2.3$)$ & $2.0(0.8$ to 3.3$)$ & $0.0(-1.3$ to 2.3$)$ & $0.0(-0.8$ to 2.3$)$ \\
\hline UPDRS 1B & $7.0(4.3$ to 8.3$)$ & $9.0(4.8$ to 11.0$)$ & $8.0(5.5$ to 9.0$)$ & $12.0(10.3$ to 12.3$)$ & $1.0(-0.3$ to 2.8$)$ & $1.0(0.0$ to 3.3$)$ \\
\hline UPDRS 2 & $7.0(5.5$ to 12.8$)$ & 12.0 (7.8 to 20.0$)$ & $8.0(5.5$ to 11.8$)$ & 14.0 (8.8 to 21.3$)$ & $0.0(-1.0$ to 2.3$)$ & $3.0(-3.0$ to 5.5$)$ \\
\hline UPDRS 3 & $21.0(14.8$ to 31.5$)$ & $24.0(15.3$ to 32.3$)$ & 25.0 (14.3 to 40.5$)$ & 28.0 (18.0 to 38.8$)$ & $1.0(-3.8$ to 4.5$)$ & $4.0(0.5$ to 14.8$)$ \\
\hline UPDRS 4 & $0.5(0.0$ to 3.5$)$ & $0.0(0.0$ to 6.0$)$ & $4.0(1.5$ to 5.0$)$ & $4.0(0.8$ to 7.3$)$ & $1.5(0.0$ to 2.5$)$ & $1.0(-0.5$ to 4.3$)$ \\
\hline PAM-13 & $70.8(66.4$ to 85.0$)$ & $56.4(47.4$ to 65.7$)$ & 70.8 (59.9 to 91.6$)$ & 63.2 (55.5 to 69.7$)$ & $0.0(-9.9$ to 4.6$)$ & $0.0(-3.7$ to 7.3$)$ \\
\hline PACIC & 3.3 (2.6 to 4.7$)$ & $3.4(2.6$ to 4.0$)$ & 3.7 (2.1 to 4.5 ) & $4.1(2.6$ to 4.4$)$ & $-0.2(-0.6$ to 0.2$)$ & $-0.1(-0.3$ to 0.6$)$ \\
\hline PDQ-39 & $12.8(11.4$ to 15.8$)$ & 21.0 (12.6 to 23.0$)$ & 17.6 (13.1 to 22.7$)$ & 22.3 (14.6 to 26.0$)$ & $5.0(-0.9$ to 10.1$)$ & $1.9(-2.0$ to 5.5$)$ \\
\hline \multicolumn{7}{|l|}{ Kinesia } \\
\hline Rest tremor & 0.1 (0.1 to 2.2 ) & $0.2(0.0$ to 1.5$)$ & 0.2 (0.1 to 1.9 ) & $0.2(0.1$ to 0.7$)$ & $0.0(-0.4$ to 0.1$)$ & $0.0(-0.3$ to 0.1$)$ \\
\hline Bradykinesia & $1.8(1.3$ to 2.4$)$ & $1.5(1.2$ to 2.0$)$ & $1.0(0.7$ to 1.6$)$ & $1.4(1.1$ to 2.0$)$ & $-0.8(-0.9 \text { to }-0.2)^{*}$ & $-0.3(-0.5$ to 0.1$)$ \\
\hline Dyskinesia & $0.1(0.0$ to 0.3$)$ & $0.1(0.0$ to 0.3$)$ & $0.1(0.0$ to 0.3$)$ & 0.1 (0.1 to 0.3$)$ & $0.0(0.0$ to 0.1$)$ & $0.0(0.0$ to 0.1$)$ \\
\hline
\end{tabular}

Values are medians and interquartile range. ${ }^{*} p=0.01$.

day was excluded from the averages, as it occurred before the individual had taken PD-related medication.

Finally, exploratory analyses examined information the neurologist gained from the motor symptom severity reports and if Kinesia could capture symptomatic changes in response to changes in medication. For participants' whose dopaminergic medication dosage was changed during the study, Kinesia metrics were correlated with a change in daily levodopa dose.

\section{Results}

Twenty-one adults were screened and 18 enrolled and randomized to control or experimental groups (Table 1). UPDRS scores, participant questionnaire responses, and Kinesia metrics were not significantly different between the groups at the start of the study $(p>0.05)$.

Time spent with the neurologist during the 3- and 6-month "visits" (in-person for the control group, videoconference or phone call for the experimental group) was significantly less for the experimental group than for the control group (29-45 min vs. 40-65 min [interquartile range], $p<0.05$ ). Table 2 shows changes from the study start to study end for UPDRS scores, patient questionnaires, and Kinesia metrics. The only outcome measure that changed during the 7-month study was bradykinesia, as measured by Kinesia, which improved for individuals in the experimental group ( $p=0.01$, Wilcoxon signed-rank test). However, this change would not be considered significant when correcting for multiple comparisons and 


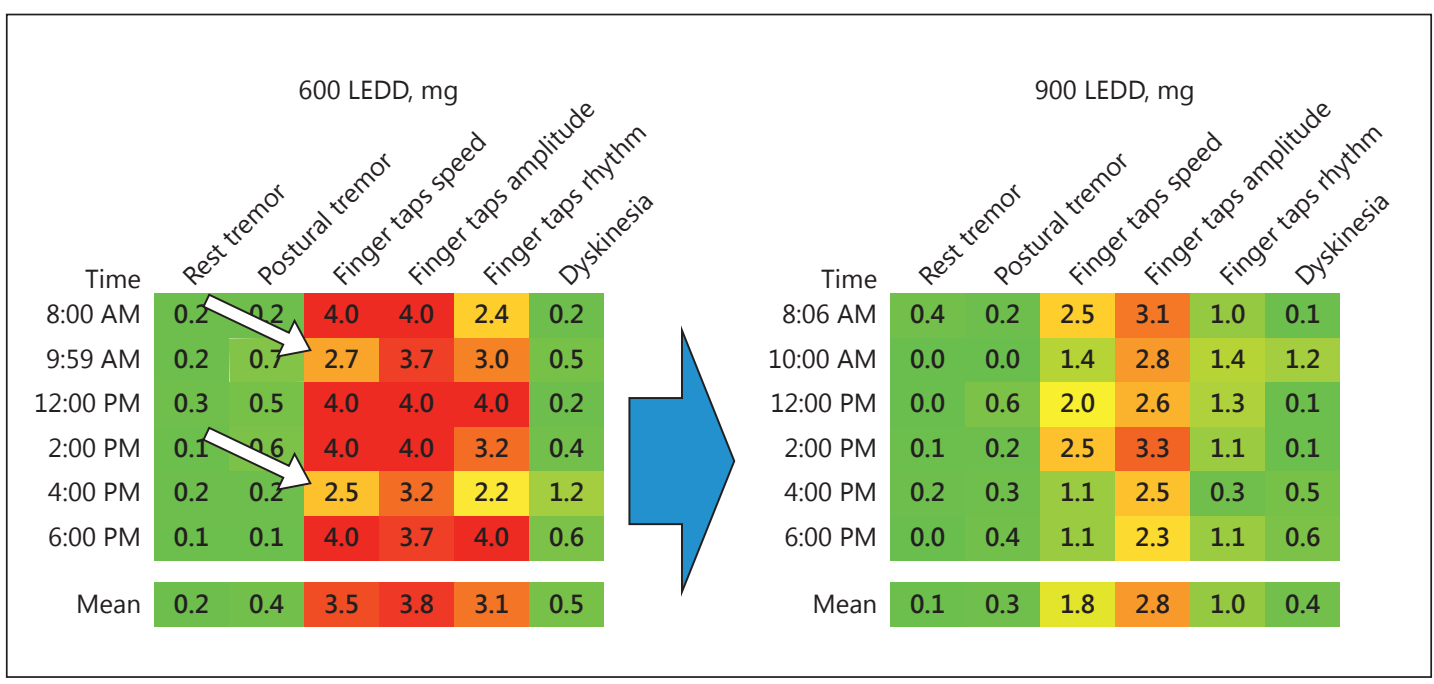

Fig. 1. Kinesia motor symptom severity report showing Parkinson's disease-related motor impairments through the day before (left) and after (right) a change in treatment. Kinesia detected improved finger-tapping speed (bradykinesia) after each dose (white arrows), prompting the neurologist to escalate therapy. Bradykinesia was significantly reduced on the higher daily levodopa dose (LEDD; $p<0.001$ ).

Fig. 2. For the 9 participants whose dopaminergic medication dosage was changed during the study, the changes in bradykinesia and dyskinesia scores measured by Kinesia are plotted versus the change in daily levodopa dose (LEDD), with decreasing Kinesia scores indicating improvement. The solid and dotted lines are the least-squares fit lines for bradykinesia $(r=-0.68, p=0.04)$ and dyskinesia $(r=-0.12, p=$ $0.77)$, respectively.

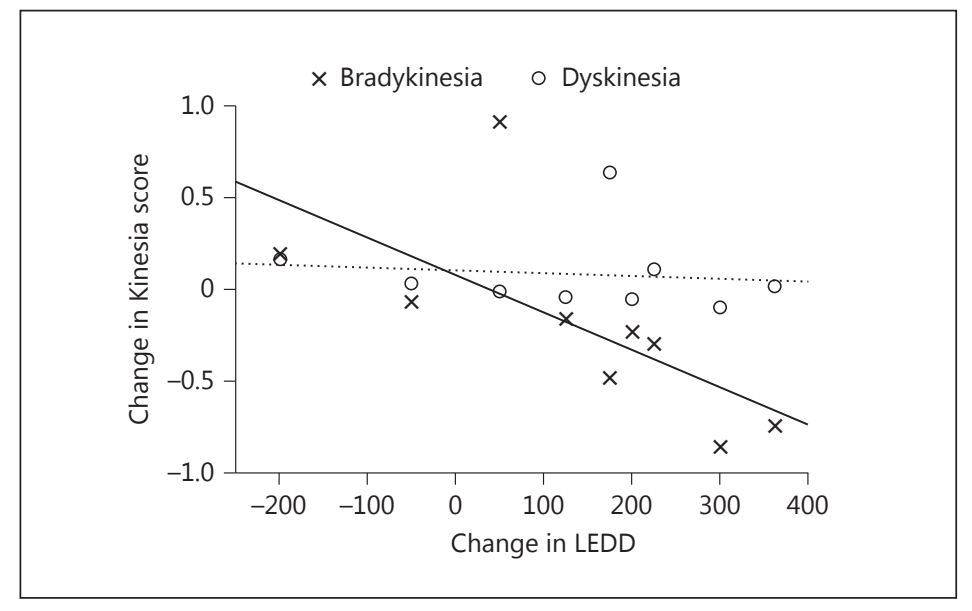

was not significant compared to improvement in the control group ( $p=0.09$, Mann-Whitney $U$ test). None of the other Kinesia metrics, UPDRS scores, or questionnaire responses changed significantly during the study nor were there any significant differences in changes between the groups.

Even though there were no significant clinometric differences between the groups, the neurologist successfully used information in the motor symptom severity reports that was not otherwise observed (e.g., levodopa responsiveness, symptoms worsening over time) to make therapy adjustments for 2 patients in the experimental group. Figure 1 shows how a report revealed a patient to be responsive to levodopa, prompting the neurologist to escalate therapy. In another instance, the reports for a young-onset patient only taking a dopamine agonist revealed symptoms to be worsening, prompting the neurologist to recommend initiating levodopa therapy. 
Table 3. Summary of Kinesia usability questionnaire responses

\begin{tabular}{|c|c|c|c|c|c|}
\hline & \multicolumn{5}{|c|}{ Likert scale } \\
\hline & 1 & 2 & 3 & 4 & 5 \\
\hline How easy was it to set up the system in your home? & $0.0 \%$ & $0.0 \%$ & $0.0 \%$ & $17.6 \%$ & $82.4 \%$ \\
\hline How easy was it to place the motion sensor on your finger? & $0.0 \%$ & $0.0 \%$ & $0.0 \%$ & $17.6 \%$ & $82.4 \%$ \\
\hline How comfortable was the motion sensor to wear? & $0.0 \%$ & $0.0 \%$ & $5.9 \%$ & $23.5 \%$ & $70.6 \%$ \\
\hline How easy were the instructions to follow and understand? & $0.0 \%$ & $0.0 \%$ & $0.0 \%$ & $17.6 \%$ & $82.4 \%$ \\
\hline How easy was it for you to use the entire system, on the whole? & $0.0 \%$ & $0.0 \%$ & $5.9 \%$ & $29.4 \%$ & $64.7 \%$ \\
\hline $\begin{array}{l}\text { How willing would you be to use this system in the future, if } \\
\text { asked by your doctor? }\end{array}$ & $0.0 \%$ & $5.9 \%$ & $11.8 \%$ & $11.8 \%$ & $70.6 \%$ \\
\hline
\end{tabular}

Questions were answered on a 1-5 Likert scale (1 = most negative, $5=$ most positive). Percentage of responses in each category are shown.

In addition to evaluating the impact of motion sensor-based telehealth diagnostics on patients with PD, we investigated if Kinesia could capture symptomatic changes in response to changes in medication. Dopaminergic medication dosage was changed during the 7-month study for 9 of the 18 study participants (4 in the experimental group and 5 in the control group). For those individuals, improvement in finger-tapping speed (bradykinesia), as measured by Kinesia, correlated with increasing daily levodopa dose $(r=-0.68, p=0.04)$; however, there was no significant correlation between change in dyskinesia severity and change in daily levodopa dose (Fig. 2).

Median compliance in using the Kinesia system at home was $95.7 \%$ with an interquartile range of 77.2-99.2\%. Seventeen of the 18 participants completed the Kinesia usability questionnaire. Responses to questions regarding overall system use are summarized in Table 3.

\section{Discussion}

In this study, individuals with PD were able and willing to use the remote monitoring technology as evidenced by the high compliance and usability questionnaire responses. The motor symptom severity reports effectively depicted symptom severities throughout the day in response to therapy and, for a subset of participants, provided actionable information to help the treating neurologist make informed therapy decisions (e.g., Fig. 1). Experience from this pilot study suggests remote monitoring will be most useful for PD patients with specific characteristics, such as individuals whose symptoms fluctuate throughout the day or those who respond to medication in a manner not observed during a clinical visit.

In addition to helping guide therapy adjustments, data captured by the remote monitoring technology showed that increases in levodopa dose correlated with improvements in bradykinesia without causing an increase in dyskinesia (Fig. 2). This suggests that medication changes were in an appropriate therapeutic range. Determining if the availability of objective evidence on how patients respond can increase clinical confidence in recommending therapy changes is a testable hypothesis worthy of further investigation.

This study was exploratory and not powered to detect differences between the groups, so it is not unexpected that none of the outcome measures were significantly different between the groups. However, the lack of required travel to the clinic and significant reduction in clinician time spent with patients, while still maintaining similar outcomes between the groups, is an important result. This has the potential to reduce healthcare costs and expand 
care access for patients who live far from movement disorder specialists. Future controlled studies, powered to detect changes in clinical metrics, will be necessary to assess if telehealth technologies improve, rather than simply maintain, patient outcomes in addition to reducing costs and patient/clinician burden.

A limitation of this study was that the experimental group's patient-clinician teleconferences were switched from videoconferences to phone calls about halfway through the study due to technical difficulties as described in the Methods section. As secure videoconferencing between patients and clinicians becomes more mainstream, it may be more practical to use third-party videoconferencing software tools alongside sensor-based assessment, rather than rely on a single system that excels at both. Other limitations include this being a singlecenter study with a relatively small sample size and the exclusion of patients with dementia. Still, the results from this pilot study could be useful for sample size calculations for largerscale multicenter studies in the future.

The Kinesia system used in this study included assessment of specific symptoms during predefined tasks rather than during spontaneous, unrestricted activities. The integration of additional sensors to measure parameters relating to gait, balance, posture, rigidity, speech, facial expression, etc., during daily activities could potentially provide a more complete picture of the patient in their natural environment. However, we must be careful to avoid using too many sensors, as they may interfere with activities, increase the burden on patients, and reduce compliance [11]. Likewise, clinicians may be less inclined to use telemedicine technologies if they must sort through large amounts of data requiring complex interpretation.

Consumer wearables linked with smartphone apps are becoming more and more common, as individuals are increasingly interested in monitoring their own health. Although this study did not include showing participants their own remotely collected data, future in-home monitoring systems will likely include patient-facing reports to encourage patient engagement. Providing patients with feedback on how their symptoms respond to therapy adjustments may make patients feel more empowered in their disease management and increase therapy compliance. Telehealth technologies including wearable sensors have the potential to reduce costs and improve care for disparate PD populations or those unable to travel.

\section{Acknowledgement}

This work was supported by the National Institute on Minority Health and Health Disparities of the National Institutes of Health under award number R44MD004049. The content of this paper is solely the responsibility of the authors and does not necessarily represent the official views of the National Institutes of Health. The authors would like to thank Maureen Phillips for assisting in study support, and Jenna Daghstani and Vanessa Zapata for providing thoughtful comments on the manuscript.

\section{Ethics Statement}

The study was reviewed and approved by the Institutional Review Board at the University of Rochester and completed in accordance with the Declaration of Helsinki. All participants provided signed informed consent prior to enrollment. 
Heldman et al.: Telehealth Management of PD

\section{Conflicts of Interest Statement}

D.A. Heldman, J.P. Giuffrida, and B. Goldberg received compensation from Great Lakes NeuroTechnologies Inc. for employment. D.A. Harris, T. Felong, K.L. Andrzejewski, E.R. Dorsey, and M.A. Burack received support from Great Lakes NeuroTechnologies Inc. through subawards from NIH/NIMHD grant number R44MD004049. E.R. Dorsey is the Editor-in-Chief of Digital Biomarkers. D.A. Heldman and J.P. Giuffrida are on the editorial board of Digital Biomarkers.

\section{References}

$>1$ Dorsey ER, George BP, Leff B, Willis AW: The coming crisis: obtaining care for the growing burden of neurodegenerative conditions. Neurology 2013;80:1989-1996.

-2 Hoffmann T, Russell T, Thompson L, Vincent A, Nelson M: Using the Internet to assess activities of daily living and hand function in people with Parkinson's disease. NeuroRehabilitation 2008;23:253-261.

-3 Samii A, Ryan-Dykes P, Tsukuda RA, Zink C, Franks R, Nichol WP: Telemedicine for delivery of health care in Parkinson's disease. J Telemed Telecare 2006;12:16-18.

4 Couzin J: Parkinson's disease. Streamlined clinical trials, from a home computer. Science 2008;320:1143.

5 Delprato U, Greenlaw R, Cristaldi M: PARKSERVICE: home support and walking aid for people with Parkinson's disease. Stud Health Technol Inform 2006;121:1-6.

6 Cubo E, Mariscal N, Solano B, Becerra V, Armesto D, Calvo S, Arribas J, Seco J, Martinez A, Zorrilla L, Heldman D: Prospective study on cost-effectiveness of home-based motor assessment in Parkinson's disease. J Telemed Telecare 2017;23:328-338.

7 Dorsey E, Vlaanderen F, Engelen L, Kieburtz K, Zhu W, Biglan K, Faber M, Bloem B: Moving Parkinson care to the home. Mov Disord 2016;31:1258-1262.

8 Papapetropoulos S, Mitsi G, Espay AJ: Digital health revolution: is it time for affordable remote monitoring for Parkinson's disease? Front Neurol 2015;6:34.

-9 Goetz CG, Tilley BC, Shaftman SR, et al: Movement Disorder Society-Sponsored Revision of the Unified Parkinson's Disease Rating Scale (MDS-UPDRS): Scale presentation and clinimetric testing results. Mov Disord 2008; 23:2129-2170.

10 Hubble JP, Pahwa R, Michalek DK, Thomas C, Koller WC: Interactive video conferencing: a means of providing interim care to Parkinson's disease patients. Mov Disord 1993;8:380-382.

11 Espay AJ, Bonato P, Nahab FB, et al: Technology in Parkinson's disease: challenges and opportunities. Mov Disord 2016;31:1272-1282.

-12 Mera TO, Burack MA, Giuffrida JP: Objective motion sensor assessment highly correlated with scores of global levodopa-induced dyskinesia in Parkinson's disease. J Parkinsons Dis 2013;3:399-407.

-13 Pulliam CL, Burack MA, Heldman DA, Giuffrida JP, Mera TO: Motion sensor dyskinesia assessment during activities of daily living. J Parkinsons Dis 2014;4:609-615.

14 Giuffrida JP, Riley DE, Maddux BN, Heldman DA: Clinically deployable Kinesia technology for automated tremor assessment. Mov Disord 2009;24:723-730.

-15 Salarian A, Russmann H, Vingerhoets FJG, Dehollain C, Blanc Y, Burkhard PR, Aminian K: Gait assessment in Parkinson's disease: toward an ambulatory system for long-term monitoring. IEEE Trans Biomed Eng 2004; 51:1434-1443.

-16 Mera TO, Filipkowski DE, Riley DE, Whitney CM, Walter BL, Gunzler SA, Giuffrida JP: Quantitative analysis of gait and balance response to deep brain stimulation in Parkinson's disease. Gait Posture 2013;38:109-114.

-17 Heldman DA, Giuffrida JP, Chen R, Payne M, Mazzella F, Duker AP, Sahay A, Kim SJ, Revilla FJ, Espay AJ: The modified bradykinesia rating scale for Parkinson's disease: reliability and comparison with kinematic measures. Mov Disord 2011;26:1859-1863.

18 Keijsers N, Horstink M, Gielen S: Ambulatory motor assessment in Parkinson's disease. Mov Disord 2006;21: 34-44.

19 Memedi M, Khan T, Grenholm P, Nyholm D, Westin J: Automatic and objective assessment of alternating tapping performance in Parkinson's disease. Sensors (Basel) 2013;13:16965-16984.

-20 Heldman DA, Jankovic J, Vaillancourt DE, Prodoehl J, Elble RJ, Giuffrida JP: Essential tremor quantification during activities of daily living. Park Relat Disord 2011;17:537-542.

21 Pulliam CL, Eichenseer SR, Goetz CG, Waln O, Hunter CB, Jankovic J, Vaillancourt DE, Giuffrida JP, Heldman DA: Continuous in-home monitoring of essential tremor. Park Relat Disord 2014;20:37-40.

22 Griffiths RI, Kotschet K, Arfon S, Xu ZM, Johnson W, Drago J, Evans A, Kempster P, Raghav S, Horne MK: Automated assessment of bradykinesia and dyskinesia in Parkinson's disease. J Parkinsons Dis 2012;2:47-55.

23 Horne MK, McGregor S, Bergquist F: An objective fluctuation score for Parkinson's disease. PLoS One 2015; $10: \mathrm{e} 0124522$. 
Heldman et al.: Telehealth Management of PD

$>24$

$>25$

$>26$

Lopane G, Mellone S, Chiari L, Cortelli P, Calandra-Buonaura G, Contin M: Dyskinesia detection and monitoring by a single sensor in patients with Parkinson's disease. Mov Disord 2015;30:1267-1271.

Arora S, Venkataraman V, Zhan A, Donohue S, Biglan KM, Dorsey ER, Little MA: Detecting and monitoring the symptoms of Parkinson's disease using smartphones: a pilot study. Park Relat Disord 2015;21:650-653.

Ferreira JJ, Godinho C, Santos AT, Domingos J, Abreu D, Lobo R, Gonçalves N, Barra M, Larsen F, Fagerbakke $\emptyset$, Akeren I, Wangen H, Serrano JA, Weber P, Thoms A, Meckler S, Sollinger S, van Uem J, Hobert MA, Maier KS, Matthew H, Isaacs T, Duffen J, Graessner H, Maetzler W: Quantitative home-based assessment of Parkinson's symptoms: the SENSE-PARK feasibility and usability study. BMC Neurol 2015;15:89.

7 Fisher JM, Hammerla NY, Rochester L, Andras P, Walker RW: Body-worn sensors in Parkinson's disease: evaluating their acceptability to patients. Telemed J E Health 2015;22:1-7.

Mera TO, Heldman DA, Espay AJ, Payne M, Giuffrida JP: Feasibility of home-based automated Parkinson's disease motor assessment. J Neurosci Methods 2012;203:152-156.

9 Dorsey ER, Venkataraman V, Grana MJ, Bull MT, George BP, Boyd CM, Beck CA, Rajan B, Seidmann A, Biglan KM: Randomized controlled clinical trial of "virtual house calls" for Parkinson disease. JAMA Neurol 2013;70: 565-570.

Biglan KM, Voss TS, Deuel LM, Miller D, Eason S, Fagnano M, George BP, Appler A, Polanowicz J, Viti L, Smith S, Joseph A, Dorsey ER: Telemedicine for the care of nursing home residents with Parkinson's disease. Mov Disord 2009;24:1073-1076.

Dorsey ER, Deuel LM, Voss TS, Finnigan K, George BP, Eason S, Miller D, Reminick JI, Appler A, Polanowicz J, Viti L, Smith S, Joseph A, Biglan KM: Increasing access to specialty care: a pilot, randomized controlled trial of telemedicine for Parkinson's disease. Mov Disord 2010;25:1652-1659.

Dalrymple-Alford JC, MacAskill MR, Nakas CT, Livingston L, Graham C, Crucian GP, Melzer TR, Kirwan J, Keenan R, Wells S, Porter RJ, Watts R, Anderson TJ: The MoCA: well-suited screen for cognitive impairment in Parkinson disease. Neurology 2010;75:1717-1725.

Peto V, Jenkinson C, Fitzpatrick R: PDQ-39: a review of the development, validation and application of a Parkinson's disease quality of life questionnaire and its associated measures. J Neurol 1998;245:S10-S14.

Glasgow RE, Wagner EH, Schaefer J, Mahoney LD, Reid RJ, Greene SM: Development and validation of the Patient Assessment of Chronic Illness Care (PACIC). Med Care 2005;43:436-444.

Hibbard JH, Mahoney ER, Stockard J, Tusler M: Development and testing of a short form of the patient activation measure. Health Serv Res 2005;40:1918-1930.

Harris PA, Taylor R, Thielke R, Payne J, Gonzalez N, Conde JG: Research electronic data capture (REDCap) - a metadata-driven methodology and workflow process for providing translational research informatics support. J Biomed Inform 2009;42:377-381.

-37 Espay AJ, Giuffrida JP, Chen R, Payne M, Mazzella F, Dunn E, Vaughan JE, Duker AP, Sahay A, Kim SJ, Revilla FJ, Heldman DA: Differential response of speed, amplitude, and rhythm to dopaminergic medications in Parkinson's disease. Mov Disord 2011;26:2504-2508.

-38 Heldman DA, Espay AJ, LeWitt PA, Giuffrida JP: Clinician versus machine: reliability and responsiveness of motor endpoints in Parkinson's disease. Park Relat Disord 2014;20:590-595. 